\title{
A study of Appointment Scheduling with No-Shows and Overbooking
}

\author{
Xudong Wang a , Runtong Zhang ${ }^{\text {b, * }}$
}

\author{
School of Economics and Management, Beijing Jiaotong University, Beijing 100044, China; \\ a15120584@bjtu.edu.cn, b11224021@bjtu.edu.cn
}

Keywords: Appointment scheduling, No-Shows, Overbooking.

\begin{abstract}
In the hospital clinic sub-time appointment mode, the patient will lead to a great waste of outpatient resources. In this paper, a multi-time multi-patient appointment strategy model is constructed in the case of patient's contract. The heuristic algorithm is used to obtain the effect of parameters such as patient's rejection rate and patient weight on optimal scheduling. We proposes an outpatient appointment strategy based on the overbooking model, which provides an appointment schedule for the hospital appointment schedule.
\end{abstract}

\section{Introduction}

There is a serious imbalance in China's medical resources supply and demand, making more and more patients went into the large public hospitals with high quality medical resourse to seek better medical services.

The appointment of treatment mechanisms avoid the large number of patients concentrated arrive at the same time, shorten the waiting time for patients to reduce the hospital congestion. For the no show behaviors, both domestic and foreign scholars have carried out research, using the overbook strategy is to reduce the positive behavior of the mainstream way [1]. In the study of the overbooking model, the study of patient characteristic variables is considered less.

Cayiri and Veral[2] have reviewed 80 papers about reservations, such as the description of outpatient appointment, evaluation of outpatient appointment system, classification and research methods. Gupta [3] discusses the opportunities and challenges in the application of reservation of resources in the hospital outpatient surgery.

Considering the patient absence under the premise of outpatient appointment optimization model has some outstanding research achievements. Sch TZ and Kolisch[5] in patients under the condition of considering the no shows, MDP model is used to optimize the hierarchical patient appointments, and through the method of approximate dynamic programming to solve the model, given a treatment cycle under the capacity allocation strategy. Based on Feldman [7] in consideration of patient preferences, cancel and miss, study how to make an appointment with more flexibility, improve patient satisfaction. The goal is to make the most daily gains. Muthuraman and Lawley[8] by means of the overflow rate, assuming that the doctor Business Hours follows an exponential distribution, a stochastic model considering the waiting time of patients, doctors and patients work overtime earnings, and numerical examples and sensitivity analysis, but the solution to the local optimal solution. BoZeng and other [9], analyze the model properties more deeply, and obtain the global optimal solution by analyzing the local optima. Chakraborty [10] is based on [8], assuming that the doctor Business Hours obey the general distribution, the overbooking model with Miss, and the depth of the mining model properties, analytical optimal solution. In China, Lan Qin [11] through the establishment of a weighted patient waiting time, queuing model is established to maximize patient satisfaction as the goal, and analyzes two kinds of reservation queuing strategy: different priority reservation queue strategy and time priority reservation queuing strategy. 


\section{Assumptions and Variables}

This model starts with the appointment scheduling staff of the hospital and schedules the patients according to the appointment of patients in the outpatient department. The following assumptions and symbolic representations of the model are presented.

A department has n number sources per day, which are assigned to $\mathrm{n}$ time periods respectively. The patient's visit time corresponds with the time period. All patients arrive at the hospital according to the scheduled time of arrival.

Assuming each patient given in reservation request, the scheduler can learn the history of miss information and patient weight information, and predict the time when patients with comprehensive coefficient $z_{j}=w_{j}\left(1-q_{j}\right) / q_{j}$. Actually, the scheduling process takes the synthetic factor as the index, and takes the minimization of the comprehensive cost as the goal. Daily doctor actual outpatient volume is $\mathrm{m}$, every day exceeds quantity $y=m-n$. The probability of reaching $r_{j}=1-q_{j}$ per patient, $J, j=1$, and $\mathrm{m}$, before the time period he was assigned, was $w$.

For each scheduling plan, the cost is divided into three parts: customer waiting cost, doctor spare cost and doctor overtime cost. Among them, when the time period $n$ starts without the arrival of the patient, the doctor will generate an idle cost of $C_{i}$, which is a waste of medical resources. At the end of the last treatment period, there are scheduling patients who need medical treatment, which will result in a doctor's overtime cost of $C_{o}$. Outpatient scheduling personnel often to reduce the waste of resources of the miss behavior by way of overbooking. If a number of customers are scheduled to arrive on time, some of the patients will be waiting. The cost of waiting for a customer $\mathrm{J}$ is $\mathrm{w}_{\mathrm{j}}$.

The probability of some patients arriving on time was $1, r_{j}=1$, and we defined this group as a set of $C_{1}$, in which the number of patients was $N_{1}<n$. Set the group $C_{2}$ indicating the punctual arrival probability of $r_{j}<1$ patients, the number of which is $N_{2}$.

It is assumed that no patient is directly arranged in the doctor's overtime period, and only during the last period of time is there a patient who has not been examined, the doctor needs to work overtime to complete the number of patients on the day of appointment.

\section{Models Design}

On the basis of the model assumptions, the horizontal- vertical scheduling model is described, and a schedule is represented as $\bar{a}=\left(a_{1}, \ldots, a_{m}\right), a_{t}$ represents the patients' number in the time period t, and the total number is

$$
\sum_{t=1}^{m} a_{t}=m \text {, and } b(n, p, k)=\left(\begin{array}{l}
n \\
k
\end{array}\right) p^{k}(1-p)^{n-k}, B^{j}\left(\bar{a}_{t}\right)=B^{j}\left(a_{1}, \ldots, a_{t}\right)
$$

represents the rate of $\mathrm{j}$ patients in the time period $\mathrm{t}$ by the schedule $\left(a_{1}, \ldots, a_{t}\right)$

$$
B^{j}\left(\bar{a}_{t}\right)=\left\{\begin{array}{cc}
B^{0}\left(\bar{a}_{t-1}\right)\left[b\left(a_{t}, r, 0\right)+b\left(a_{t}, r, 1\right)\right]+B^{1}\left(\bar{a}_{t-1}\right) b\left(a_{t}, r, 0\right) & \text { for }=0 \\
\sum_{i=0}^{l\left(\bar{a}_{t-1}\right)} B^{i}\left(\bar{a}_{t-1}\right) b\left(a_{t}, r, j-i+1\right) & \text { for } 1 \leq j \leq l\left(\bar{a}_{t}\right)
\end{array}\right.
$$

$l\left(\bar{a}_{t}\right)=\sum_{i=1}^{t} a_{i}-t$ represents the max number of patients in time period $\mathrm{t}$.

If a patients scheduled in the same time period, and three are $\mathrm{k}$ patients waiting in the queue, $W(a, k)$ represents the total waiting time expectation value of the a patients:

$$
W(a, k)=r \sum_{i=1}^{a} \sum_{j=0}^{i-1}(k+j) b(i-1, r, j)
$$

In the scheduling $\bar{a}$, the total waiting time is:

$$
W(\bar{a})=\sum_{t=1}^{T} \sum_{j=0}^{l\left(\bar{a}_{t-1}\right)} B^{j}\left(\bar{a}_{t-1}\right) W\left(a_{t}, j\right)
$$

The doctor's free period of time can be expressed as: 


$$
I(\bar{a})=\sum_{t=1}^{n} B^{0}\left(\bar{a}_{t-1}\right) b\left(a_{t}, r, 0\right)
$$

Oa indicates the expected value of the number of overtime periods, if no patient is scheduled in overtime, $\mathrm{T}=\mathrm{n}, O(\bar{a})=\sum_{j=0}^{l\left(\bar{a}_{n}\right)} j B^{j}\left(\bar{a}_{n}\right)$, when $\mathrm{T}>\mathrm{n}$, Overtime time there will be three cases, the first case, all arranged in period $\mathrm{T}$ patients no show, and has no rest of the patients before the time period, doctors will work T-n-1 overtime period, second cases, only one patient arrive at time $\mathrm{T}$, the doctor will work T-n overtime period. In the third case, the overtime period is T-n, plus the number of patients remaining in the previous period. Therefore

$$
\begin{aligned}
& O(\bar{a})=(T-n-1) B^{0}\left(\bar{a}_{T-1}\right) b\left(a_{T}, r, 0\right) \\
& +(T-n)\left[B^{0}\left(\bar{a}_{T-1}\right) b\left(a_{T}, r, 1\right)+B^{1}\left(\bar{a}_{T-1}\right) b\left(a_{T}, r, 0\right)\right] \\
& +\sum_{j=1}^{l\left(\bar{a}_{T}\right)}(T-n+j) B^{j}\left(\bar{a}_{T}\right) \\
& =\sum_{j=0}^{l\left(\bar{a}_{T}\right)}(T-n+j) B^{j}\left(\bar{a}_{T}\right)-B^{0}\left(\bar{a}_{T-1}\right) b\left(a_{T}, r, 0\right), T>n
\end{aligned}
$$

The optimal scheduling $\bar{a}^{*}=\left(\bar{a}_{1}^{*}, \ldots, \bar{a}_{m}^{*}\right)$ is optimum solution of

$$
V^{*}=\min _{\bar{a}}\left\{V(\bar{a})=c_{I} I(\bar{a})+w W(\bar{a})+c_{O} O(\bar{a})\right\}
$$

By calculating, we can obtain the optimal solution for the scheduling.

\section{Optimal Schedules}

Considering the complexity of the model, too many variables. We uses the MATLAB software, setting the numerical parameters and using the software of enumeration algorithm, analysis the optimal relationship between scheduling and parameters.

This model is mainly to solve a department scheduling problem, because the doctor overtime costs, patients waiting cost is not the key variables in the following discussion, according to the research results of Christos Zacharias and Michael Pinedo [14], were studied by the data in the paper. In the conventional model, considering the negative factors existing in the doctor's overtime work, the working cost coefficient $C_{o}=1.5$ is set up under the condition that the normal work cost is the ratio coefficient 1 . Patient waiting cost factor $\mathrm{w}, 0<w<1$. Set normal daily hours of $n=12$.

The target cost function input calculation software MATLAB, set general variables $w, q$, and a certain period of time, the number of $n$ services and the number of $y$, through the MATLAB traversal methods changing the parameters, the calculation of target cost function under the condition of minimum, and we calculate the specific parameters of the optimal scheduling results.

Through the calculation of MATLAB, a list of different waiting cost coefficient $\mathrm{w}$ and the patients no show probability for the $q$ case, the optimal variation of parameters under the condition of scheduling results. For example, in the first row, assuming the number of patients in the diagnosis and treatment of $y=14$, diagnosis and treatment of patients with $n=12$ interval number, weight $\mathrm{w}=0.01$, with average $q=0.2$ no show rate case, the optimal scheduling model is the first time for the 3 patients, after each time for 1 patients.

It can be seen from table 1, the overbooking y decreased with the increase of customer waiting cost, increased with increasing miss probability. At the same time, you can see most of the overbooking period is getting ahead. Changes in the actual arrangement of the number of $m$ patients with the no show rate and wait for weight change, and is not a fixed value. 
Table 1 Parameter $w$ and $q$ change optimal scheduling results table

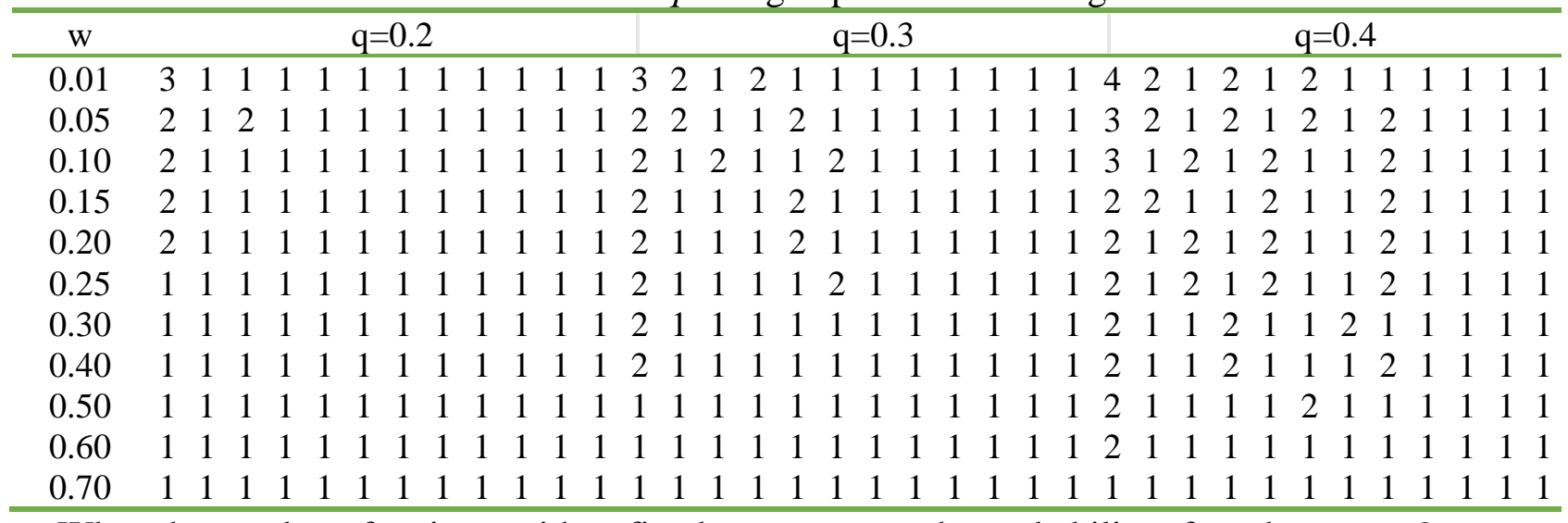

When the number of patients with m fixed arrangement, the probability of no show rate $Q$, we can see the relationship between the optimal scheduling results and no show rate. The table shows the schedule of the minimum target cost when the $w=0.1$ and $m=18$ are fixed. It can be seen that when the $q$ no show rate is very small, the doctor overtime relative increase in overtime costs increased significantly, and almost no overbooking, beyond the total outpatient patients are directly arranged in the overtime period. When the no show rate is gradually increased, the doctor overtime is gradually reduced, and corresponding overbooking situation. When the no show rate is higher than 0.4, overbooking situation increased significantly, and the beginning time of the forward overbooking.

Table 2 Fixed patient number $m=18$ optimal schedule

\begin{tabular}{cccccccccccccccccc}
\hline $\mathrm{q}$ & \multicolumn{11}{c}{ OORMAL } \\
\hline 0.000 & 1 & 1 & 1 & 1 & 1 & 1 & 1 & 1 & 1 & 1 & 1 & 1 & 1 & 1 & 1 & 1 & 1 \\
0.050 & 2 & 1 & 1 & 1 & 1 & 1 & 1 & 1 & 1 & 1 & 1 & 1 & 1 & 1 & 1 & 1 & \\
0.100 & 2 & 1 & 1 & 1 & 1 & 1 & 2 & 1 & 1 & 1 & 1 & 1 & 1 & 1 & 1 & \\
0.125 & 2 & 1 & 1 & 1 & 2 & 1 & 1 & 1 & 2 & 1 & 1 & 1 & 1 & 1 & 1 & \\
0.150 & 2 & 1 & 1 & 2 & 1 & 1 & 1 & 2 & 1 & 1 & 1 & 1 & 1 & & \\
0.200 & 2 & 2 & 1 & 1 & 2 & 1 & 1 & 1 & 2 & 1 & 1 & 2 & & & \\
0.250 & 2 & 2 & 1 & 2 & 1 & 2 & 1 & 1 & 2 & 1 & 2 & 1 & & & \\
0.300 & 3 & 1 & 2 & 1 & 2 & 1 & 2 & 1 & 1 & 2 & 1 & 1 & & & \\
0.350 & 3 & 1 & 2 & 1 & 2 & 1 & 2 & 1 & 2 & 1 & 1 & 1 & & & \\
0.400 & 3 & 2 & 1 & 2 & 1 & 2 & 1 & 2 & 1 & 1 & 1 & 1 & & & \\
0.500 & 3 & 1 & 2 & 2 & 1 & 2 & 1 & 2 & 1 & 1 & 1 & 1 & & & \\
0.600 & 2 & 2 & 2 & 1 & 2 & 2 & 1 & 2 & 1 & 1 & 1 & 1 & & & \\
0.700 & 2 & 1 & 1 & 1 & 1 & 1 & 1 & 2 & 1 & 1 & 1 & 1 & & & \\
0.800 & 2 & 2 & 1 & 2 & 2 & 1 & 2 & 1 & 2 & 1 & 1 & 1 & & & \\
\hline
\end{tabular}

Because of the computational complexity, the optimal overbooking level is difficult to produce analytically. This paper uses regression analysis model, through data analysis, obtained the relationship between overbooking level y and independent variables $n, Q, W$. At this point, there is still an idle cost of $C_{O}=1.5,0<\mathrm{W}<1$. We can calculate a large amount of overbooking between y and different $n, q, w$ through MATLAB. According to the literature Greene2008[13], we can see that there is a Poisson regression between $\mathrm{y}$ and independent variables $n, q, w, y=e^{\beta_{1} n+\beta_{2} w+\beta_{3} q+\beta_{4}}$, and $\bar{\beta}=(0.15,-2.70,8.36,-3.26)$, in which the optimal overbooking level can be calculated.

\section{Summary}

We study an overbooking model for scheduling the arrivals of patients who have different no-show probabilities and different weights. We explore the trade-offs between the benefits of efficient physician utilization and the costs of patients' waiting time. This paper proposes an outpatient reservation strategy based on overbooking model, which provides an alternative scheme 
for hospital reservation scheduling. A more accurate scheduling strategy is developed with full consideration of each patient's characteristics.

In order to simplify the computation of the model, the enumeration method is used to solve the model. This method is only suitable for small scale scheduling, and the efficiency of the solution is very low when the model variables are enlarged. The next step is to optimize the model, achieve mathematical analysis, and obtain more adaptive results.

\section{Acknowledgments}

This work was partially supported by a Key Project of National Natural Science Foundation of China (NSFC) with grant number 71532002.

\section{References}

[1]. Feng Liang, Ningbo Wang, Cheng-you Shi, et al. Analysis of Management Problems in Outpatient Appointment System. Vol. 35(2015) No. 9, p.28-31.

[2]. Cayirli T, Veral E. Outpatient Scheduling in Health care: A Review of Literature. Production \& Operations Management. Vol. 12(2003) No. 4, p.519-549.

[3]. Diwakar Gupta, Brian Denton. Appointment scheduling in health care: Challenges and opportunities. IIE Transactions. Vol. 40(2008) No. 9, p.800-819.

[4]. Gupta D, Wang L. Revenue Management for a Primary-Care Clinic in the Presence of Patient Choice. Operations Research. Vol. 56(2008) No. 3, p.676-592.

[5]. Hans-Jörg Schütz, Kolisch R. Approximate dynamic programming for capacity allocation in the service industry. European Journal of Operational Research. Vol. 218(2102) No. 1, p.239-250.

[6]. Angst C, Devaraj S, Queenan C, Greenwood B. Performance effects related to the sequence of integration of healthcare technologies. Production and Operations Management. Vol. 2101(20) No. 3, p.319-333.

[7]. Feldman J, Liu N, Topaloglu H, et al. Appointment Scheduling Under Patient Preference and No-Show Behavior. Operations Research. Vol. 62(2014) No. 4, p.794-811.

[8]. Kumar Muthuraman, Mark Lawley. A stochastic overbooking model for outpatient clinical scheduling with no-shows. IIE Transactions. Vol. 40(2007) No. 9, p. 44338-44346.

[9]. Zeng B, Turkcan A, Lin J, et al. Clinic scheduling models with overbooking for patients with heterogeneous no-show probabilities. Annals of Operations Research. Vol. 178(2010) No. 1, p. 121-144.

[10]. Santanu Chakraborty, Kumar Muthuraman, Mark Lawley. Sequential clinical scheduling with patient no-shows and general service time distributions. IIE Transactions. Vol. 42(2010) No.5, p. 354-366.

[11]. Lan Qin, Yinfeng Xu. Research of Scheduling Outpatient Appointments Based on Patients Satisfaction. Operations Research and Management Science. Vol.22 (2013), No. 2, p. 34-42.

[12]. Pingping Cao, Jiafu Tang. Capacity Allocation Policy of Outpatient Appointment Considering Cancellation of Appointment. Operations Research and Management Science. Vol.23 (2014), No.2, p. 2144.

[13]. Delaurentis P C, Kopach R, Rardin R, et al. Open Access Appointment Scheduling-An Experience At A Community Clinic. Operations Research and Management Science. Vol.23 (2006), No.2, p. 231-244.

[14]. Zacharias C, Pinedo M. Appointment Scheduling with No-Shows and Overbooking. Production \& Operations Management. Vol.23 (2014), No.5, p.788-801. 